\title{
Temperature-dependent viscosity analysis of SAE 10W-60 engine oil with RheolabQC rotational rheometer
}

\author{
Dănuț Zahariea ${ }^{1, *}$, and Dorin Emil Husaru ${ }^{2}$ \\ 1"Gheorghe Asachi" Technical University of Iași, Department of Fluid Mechanics, Fluid Machinery \\ and Fluid Power Systems, D. Mangeron 59A, Iași, România \\ 2"Gheorghe Asachi" Technical University of Iași, Department of Mechanical Engineering, \\ Mecatronics and Robotics, D. Mangeron 43, Iași, România
}

\begin{abstract}
The purpose of this work was to determine a viscositytemperature relationship for SAE $10 \mathrm{~W}-60$ engine oil. The rheological properties of this engine oil, for a temperature range of $20 \div 60{ }^{\circ} \mathrm{C}$, were obtained with RheolabQC rotational rheometer. For the first reference temperature of $40{ }^{\circ} \mathrm{C}$, the experimental result was obtained with a relative error of $1.29 \%$. The temperature-dependent viscosity was modelled, comparatively, with the Arrhenius and the $3^{\text {rd }}$ degree polynomial models. Comparing the graphs of the fits with prediction bounds for $95 \%$ confidence level, as well as the goodness-of-fit statistics, the preliminary conclusion was that the $3^{\text {rd }}$ degree polynomial could be the best fit model. However, the fit model should be used also for extrapolation, for the second reference temperature of $100^{\circ} \mathrm{C}$. This new approach changes the fit models order, the Arrhenius equation becoming the best fit model, because of the completely failed to predict the extrapolated value with the polynomial model.
\end{abstract}

\section{Introduction}

The liquids viscosity is very sensitive to the temperature, especially for different kind of engine oils. Generally, for any fluid viscosity analysis, the corresponding temperature should be mentioned. There are many temperature-dependent viscosity models, many of these for a specific class of liquids; one of the most well-known being the Arrhenius equation. For a certain liquid, it is recommended to obtain by experiment the viscosity at different temperatures and then, to find the values of the parameters from the Arrhenius equation which fits the experimental data. This methodology was used in many researches, for analysis of different liquids: biodiesel fuels [1], vegetable oils [2], vegetable oil-diesel fuels blends [3], and pollock oil [4]. Different other temperature-dependent viscosity models were also used, for example $3^{\text {rd }}$ degree polynomial models for blends of soybean oil, soy biodiesel and petroleum diesel oil [5], Vogel equation, $6^{\text {th }}$ degree polynomial and Gaussian equation for $10 \mathrm{~W}-40$ engine oil [6], $3^{\text {rd }}$ and $4^{\text {th }}$ degree polynomial for $0 \mathrm{~W}-40,5 \mathrm{~W}-40,10 \mathrm{~W}-40,15 \mathrm{~W}-40$ and 20W-40 engine oils [7].

\footnotetext{
*Corresponding author: dzahariea@yahoo.com
} 
One of the most important measurement method of rheological properties of fluids is the rotational method which consists, in principal, in two parts: one volume calibrated cup which will be filled with a precise volume of sample fluid and a measuring bob having a certain geometry. The Searle rotational method assume stationary calibrated cup and rotational bob. On the contrary, the Couette rotational method assume rotational cup and stationary measuring bob, [8]. For the Searle rotational method, the measuring bob will be driven with a controlled rotational speed $n[\mathrm{rpm}]$, as consequence the shear rate $\dot{\gamma}[1 / \mathrm{s}]$ will be known. The measured parameter is the shaft torque $M[\mathrm{Nm}]$, as consequence the shear stress $\tau[\mathrm{Pa}]$ will be known, and finally the dynamic viscosity $\eta$ [Pas] will be compute with the formula $\eta=\tau / \dot{\gamma}$. This is the so called controlled shear test, CSR. There is also another type of Searle rotational method, known as the controlled shear stress test, CSS, which consists in setting the torque/shear stress, measuring the rotational speed/shear rate, and finally obtaining the dynamic viscosity, [9]. Depending on the geometry, the rotational rheometers may be: concentric cylinder measuring system (CC MS); double-gap measuring system (DG MS); combined cylinder and cone-and-plate measuring system (Mooney/Ewart measuring system, ME MS); cone-and-plate measuring system (CP MS) and parallel-plate measuring system (PP MS), [9].

In this study, the RheolabQC rotational rheometer with concentric cylinder (CC MS) [10] will be used for performing a CSR test in order to evaluate the rheological properties of the Castrol EDGE SAE $10 \mathrm{~W}-60$ engine oil for a temperature range of $20 \div 60^{\circ} \mathrm{C}$. The experimental data will be validated considering the viscosity presented in the Castrol EDGE SAE $10 \mathrm{~W}-60$ data sheet for the first reference temperature of $40^{\circ} \mathrm{C}$, [11]. Two temperaturedependent viscosity models will be used: the Arrhenius model, and the $3^{\text {rd }}$ degree polynomial. In order to obtain the best fit model a comparatively analysis will be performed from the point of view of the graphs of the fits with prediction bounds for $95 \%$ confidence level, as well as of the goodness-of-fit statistics. However, the final decision will depend also on the viscosity obtained by extrapolation for the second reference temperature of $100^{\circ} \mathrm{C}$.

\section{Rheometer description}

RheolabQC is a rotational rheometer composed by the main components: 1-basic unit, 2coupling for connecting measuring systems, 3-temperature control unit, 4-measuring systems, and 5-computer with Rheoplus software, Figure 1.

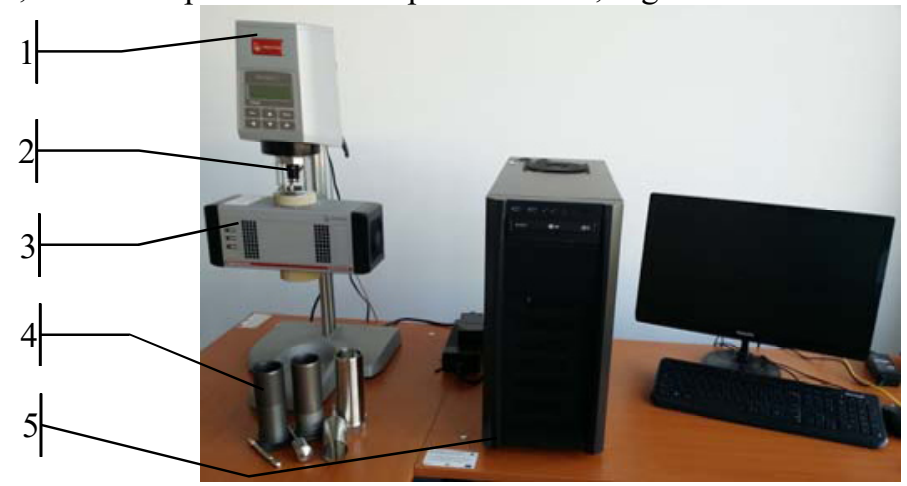

Fig. 1. RheolabQC rotational rheometer-general view.

The basic unit contain the rheometer principal components: mechanics, electronics and control systems and can be directly operated using a keypad and a LCD display from the front view of the basic unit, Figure 2, a). On the rear view of the basic unit there are different connectors: power supply, RS232 for PC or printer, Ethernet, PS2 for keyboard, Pt100 
temperature sensor, Figure 2, b). The coupling for connecting the measuring systems is presented in Fig. 2, c). The coupling includes also an automatic component recognition and configuration system, named Toolmaster, for taking into considerations and transferring to the Rheoplus software all the relevant information about the connected measuring system. The relevant data is stored in a transponder chip in each measuring system. The air countercooling Peltier temperature control unit is presented in Figure 2, d). The temperature can be adjusted in the range of $0 \div 180^{\circ} \mathrm{C}$. Three measuring systems can be used, Figure 2, e): two concentric cylinder measuring systems CC10, Figure 2, f) and CC27, Figure 2, g); and one double-gap measuring system DG42, Figure 2, h).

The RheolabQC principal technical specifications are: viscosity measuring range $1 \div 10^{9}$ mPas; rotational speed $0.01 \div 1200 \mathrm{rpm}$; torque $0.25 \div 75 \mathrm{mNm}$; shear stress $0.6 \div 17800 \mathrm{~Pa}$; shear rate $0.02 \div 40001 / \mathrm{s}$.

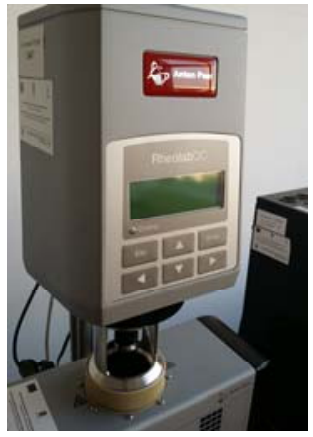

a) basic unit front view

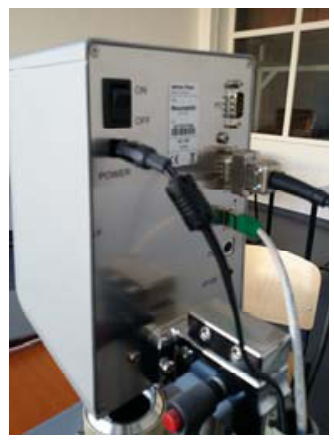

b) basic unit rear view

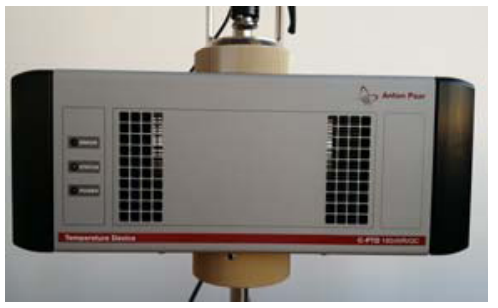

d) temperature control unit

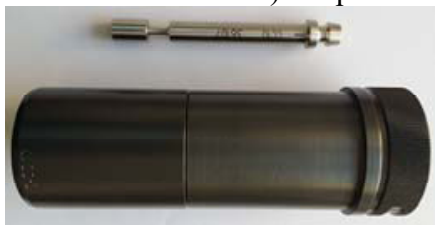

f) concentric cylinder measuring system $\mathrm{CC} 10$

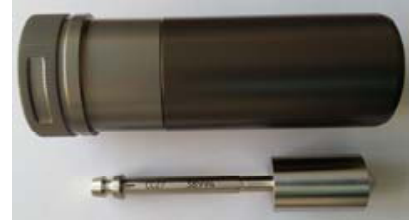

g) concentric cylinder measuring system $\mathrm{CC} 27$

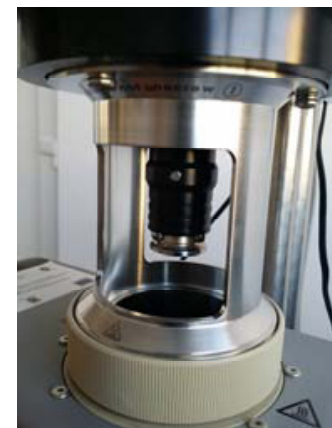

c) coupling for connecting the measuring systems

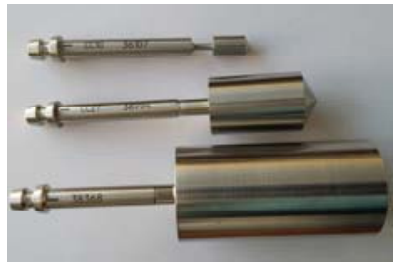

e) measuring systems

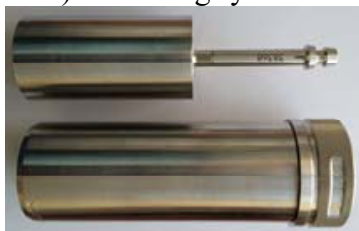

h) double-gap measuring system DG42

Fig. 2. RheolabQC rotational rheometer-main components.

\section{Setting up the experiment}

For this experiment, the CC27 concentric cylinder measuring system has been selected. The sample volume for this measuring system is $19 \mathrm{ml}$, thus the measuring system has been filled with this volume of SAE 10W-60 oil. Then, the sample container was push up through the Peltier temperature control unit and locked up with the locking screw, meanwhile the measuring system was connected with the RheolabQC coupling system. The experiment was controlled using the Rheoplus software with a CSR flow curve default workbook. The 
measuring system (CC27-SN38994) has been detected using the Toolmaster automatic component recognition and configuration system. The principal characteristics of CC27SN38994 measuring system are: maximum shear rate $1935.96 \mathrm{~s}^{-1}$ and maximum shear stress 952.311 Pa. For the first experiment, the setup temperature is $20^{\circ} \mathrm{C}$.

The measuring profile is defined by the linear ramp shear rate range $\dot{\gamma}=1 \div 100 \mathrm{~s}^{-1}$, with 50 measuring points and a linear variable measuring point duration starting from 5 seconds and ending to 10 seconds, thus the total experiment interval being 375 seconds. Before starting the first test, a temperature control process was defined with the setup temperature of $20^{\circ} \mathrm{C}$ and the temperature tolerance of $\pm 0.1^{\circ} \mathrm{C}$, with 2-minute checking time and 5-minute timeout.

\section{Experimental results}

The first experiment has been carried out at the temperature of $20^{\circ} \mathrm{C}$. The experimental data is presented in Figure 3, a) for the flow curve and Figure 3, b) for the viscosity curve. The flow curve suggests the newtonian character of the sample. In order to aproximate the experimental viscosity curve, the Newton model function (linear regression method) has been used, which represent the recommended method for idealviscous flow behavior. The experimental data and the Newton regression line are presented in Figure 3, c); the approximated value of dynamic viscosity being $364.59 \mathrm{mPas}$. Several new experiments have been performed using the same methodology and the same parameters, except the temperature for which the following values have been considered: $30^{\circ} \mathrm{C}, 40^{\circ} \mathrm{C}, 50^{\circ} \mathrm{C}$ and $60^{\circ} \mathrm{C}$. The approximated values of dynamic viscosity obtained with the same Newton reggresion method are presented in Figure 4.

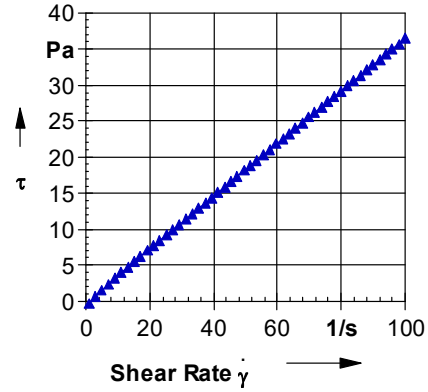

a) flow curve

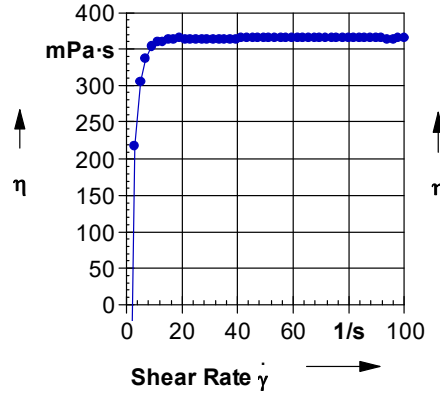

b) viscosity curve

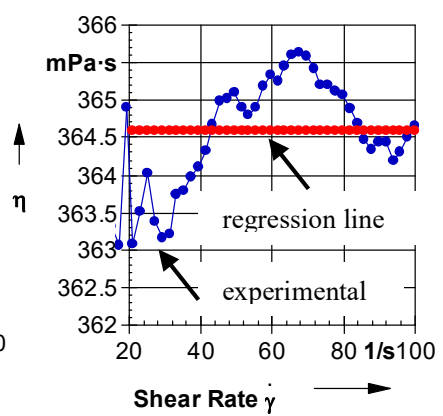

c) viscosity curve-linear regression

Fig. 3. Experimental data and Newton regression approximation for $\mathrm{t}=20^{\circ} \mathrm{C}$.

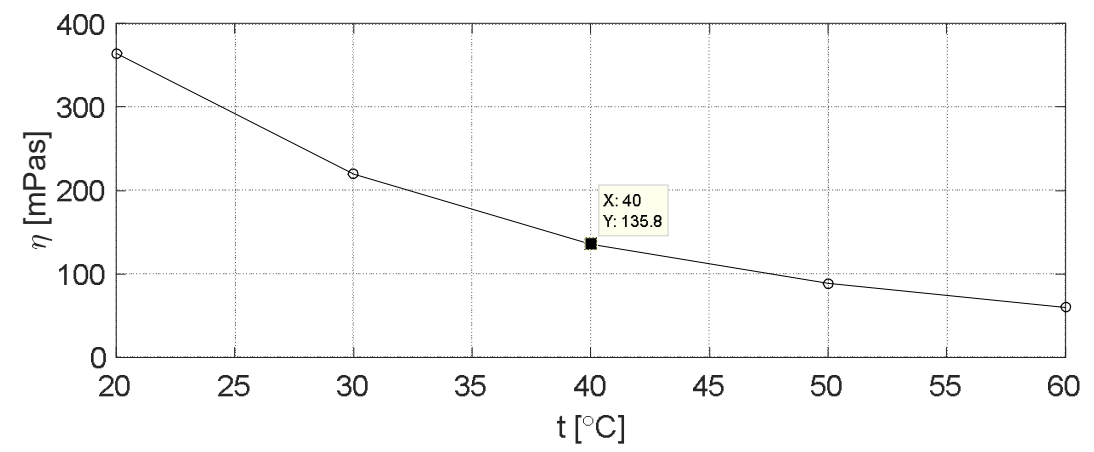

Fig. 4. Approximated dynamic viscosity. 
In order to validate the experimental data obtained with RheolabQC rheometer and presented Figure 4, the information provided in the SAE $10 \mathrm{~W}-60$ product data sheet has been considered, [11]. According to this data sheet, at $40^{\circ} \mathrm{C}$ the kinematic viscosity is $160 \mathrm{~mm}^{2} / \mathrm{s}$. At the same temperature, considering the density $0.838 \mathrm{~g} / \mathrm{cm}^{3}$ from [8] and the dynamic viscosity $135.81 \mathrm{mPas}$ from Figure 4, the kinematic viscosity will be $162.06 \mathrm{~mm}^{2} / \mathrm{s}$, for which the relative error respect to the product data sheet value is only $1.29 \%$.

\section{Discussion}

In order to obtain a temperature-dependent viscosity relationship, the Arrhenius model has been used comparatively with the $3^{\text {rd }}$ degree polynomial model. The equations, the coefficients and the goodness-of-fit statistics (the sum of squares due to error-SSE, R-square, Adjusted R-square, the root mean squared error-RMSE) are presented in Table 1. The graphs of the fits with prediction bounds for 95\% confidence level are presented in Figure 5 (Arrhenius model), and Figure 6 (polynomial model). From Table 1, it is obvious that the polynomial model leads to better goodness-of-fit statistics for all parameters; thus it would seem that the polynomial model would be the answer. Moreover, for the experimental temperature range $20 \div 60^{\circ} \mathrm{C}$, there are not significant discrepancies related to the shape of the fit curves, including the prediction bounds. Outside of the experimental temperature range, however, is something else; the polynomial curve change dramatically his shape, together with the prediction bounds which are strongly diverging. The estimated viscosity for the second reference temperature is $-302.2 \mathrm{mPas}$; the polynomial model completely failed to predict this value (which is $18.17 \mathrm{mPas},[11]$ ).

Table 1. Temperature-dependent viscosity model's characteristics.

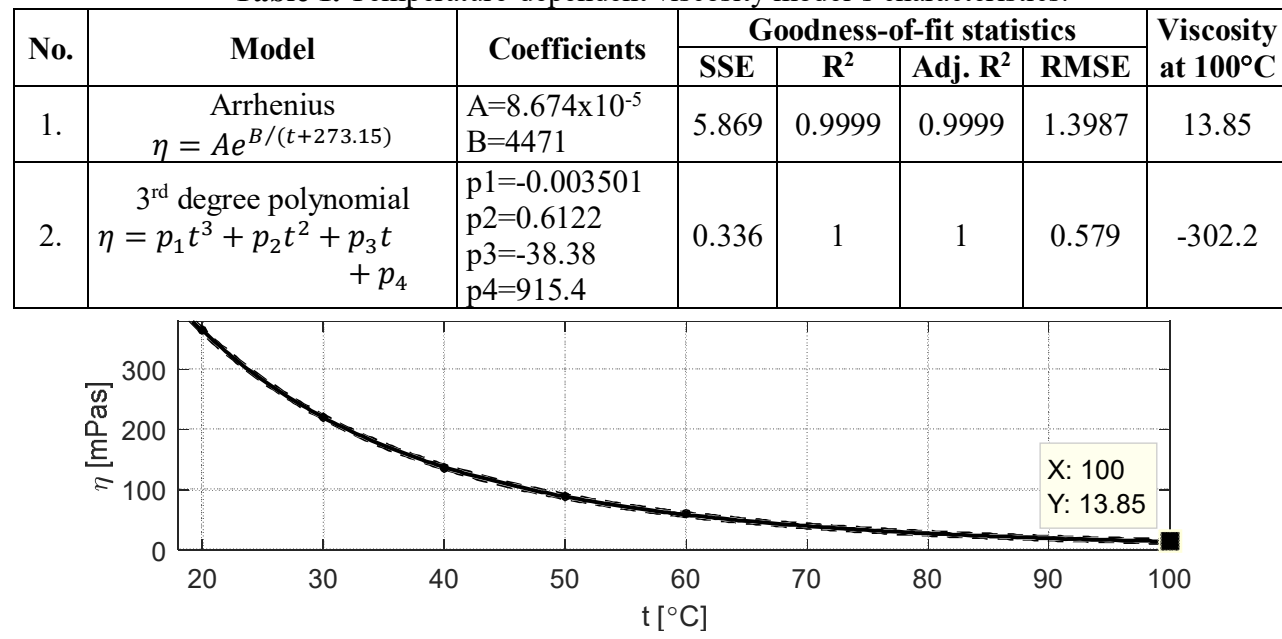

Fig. 5. Fit curve with prediction bounds, confidence level 95\%-Arrhenius model.

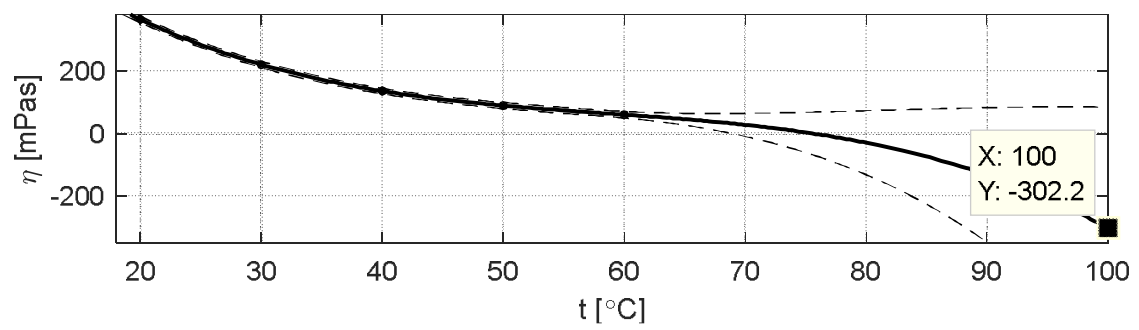

Fig. 6. Fit curve with prediction bounds, confidence level $95 \%-3^{\text {rd }}$ degree polynomial model. 
On the other side, for the Arhenius model, a very good behaviour can be observed outside of the temperature range, the prediction bounds are quite uniform, and the estimated viscosity for $100^{\circ} \mathrm{C}$ is $13.85 \pm 4.55 \mathrm{mPas}$, with confidence level of $95 \%$, which is a very good result, considering the large temperature range $60 \div 100^{\circ} \mathrm{C}$ without experimental data.

\section{Conclusions}

RheolabQC rotational rheometer can be successfully used for temperature-dependent viscosity experimental analysis. The fluid viscosity is extremely sensitive at the temperature; thus, a high-performance temperature control unit (like the Peltier unit C-LTD180/QC) is mandatory for rheological experiments. The rheological test performed on the Castrol EDGE SAE 10W-60 engine oil shows a good agreement with the product data, with a relative error of $1.29 \%$ at the first reference temperature of $40^{\circ} \mathrm{C}$.

Comparing multiple fit models should be done not only by comparing the goodness-offit statistics; in fact, for this analysis all these coefficients indicate that the polynomial model should be preferred. However, considering the ability of the model to estimate outside of the experimental temperature range, it has been proven that this first conclusion was wrong; the Arrhenius model being capable to estimate the product data sheet viscosity value of 18.17 $\mathrm{mPas}$ with the interval $13.85 \pm 4.55 \mathrm{mPas}$, with confidence level of $95 \%$, while the polynomial model with the estimated value of $-302.2 \mathrm{mPas}$ represents a total failure. Improving the accuracy of the fit model can be done by increasing the number of experimental data points, inside the initial temperature range $20 \div 60^{\circ}$, but also, if it is possible, for higher temperatures.

The authors would like to acknowledge the technical resources offered by the Laboratory of Fluid Properties Measurement, from the Department of Fluid Mechanics, Fluid Machinery and Fluid Power Systems, "Gheorghe Asachi" Technical University of Iasi, Romania. The Laboratory of Fluid Properties Measurement has been equipped with technical resources with the financial support of the grant ENERED, POSCCE-A2-O2.2.1-2009-4, ID 911.

\section{References}

1. S. Kerschbaum, G. Rinke, Fuel 83 (3) 287-291 (2004)

2. S.G.E. Giap, J. Phys. Sci. 21 (1), 29-39 (2010)

3. Z. Franco, Q.D. Nguyen, Fuel 90 (2), 838-843 (2011)

4. S. Sathivel, J. Huang, W. Prinyawiwatkul, J. Food Eng. 84 (2), 187-193 (2008)

5. O.A. Aworanti, S.E. Agarry, A.O. Ajani, Adv. Chem. Eng. Sci. 2, 444-452 (2012)

6. L. Severa, M. Havlicek, V. Kumbar, Acta Univ. Agric. Silvic. Mendel. Brun. 4, 95-102 (2009)

7. V. Kumbar, A. Sabaliauskas, Acta Univ. Agric. Silvic. Mendel. Brun., 6, 95-102, $1763-$ 1767 (2013)

8. http://www.viscopedia.com

9. T.G. Mezger, The Rheology Handbook. For users of rotational and oscillatory rheometers (Vincentz Network, Hannover, 2006)

10. http://www.anton-paar.com/us-en/products/details/quality-control-rheometerrheolabqc/

11. http://msdspds.castrol.com/bpglis/ FusionPDS.nsf/Files/33478B845E8AACDD80257FDB004B749E/\$File/BPXEABN7DR.pdf 